\title{
Successful treatment of COVID-19-related acute respiratory distress syndrome with a rare blood type: A case report
}

\author{
Hiroyuki Yamada $^{1,2}$ (1) Shigeru Ohtsuru ${ }^{1}$ | Mika Nagatomo ${ }^{1}$ | Yohei Korogi ${ }^{3}$ | \\ Ken Shinozuka ${ }^{1}$ | Naoya Tanabe ${ }^{3}$ | Shinichi Kai ${ }^{4}$ | Takeshi Matsubara ${ }^{2}$ | Isao Ito $^{3}$ | \\ Masahiro Ihara $^{5}$ | Tomoharu Tanaka ${ }^{4}$ Genta Kato ${ }^{6} \mid$ Miki Nagao $^{7}$ | Hiroshi Date ${ }^{8}$ \\ ${ }^{1}$ Department of Primary Care and Emergency Medicine, Graduate School of Medicine, Kyoto University, Kyoto, Japan \\ ${ }^{2}$ Department of Nephrology, Graduate School of Medicine, Kyoto University, Kyoto, Japan \\ ${ }^{3}$ Department of Reparatory Medicine, Graduate School of Medicine, Kyoto University, Kyoto, Japan \\ ${ }^{4}$ Department of Anesthesia, Graduate School of Medicine, Kyoto University, Kyoto, Japan \\ ${ }^{5}$ Department of Medical Informatics, Graduate School of Medicine, Kyoto University, Kyoto, Japan \\ ${ }^{6}$ Solutions Center for Health Insurance Claims, Kyoto University Hospital, Kyoto, Japan \\ ${ }^{7}$ Department of Clinical Laboratory Medicine, Graduate School of Medicine, Kyoto University, Kyoto, Japan \\ ${ }^{8}$ Department of Thoracic Surgery, Graduate School of Medicine, Kyoto University, Kyoto, Japan
}

\section{Correspondence}

Shigeru Ohtsuru, 54 Shogoin-

kawaharacho, Sakyo-ku, Kyoto

6068507, Japan.

Email: ohtsuru@kuhp.kyoto-u.ac.jp

Funding information

None

\begin{abstract}
Extracorporeal membrane oxygenation is indispensable for critically severe COVID-19 patients. However, it would be inapplicable to patients with a rare blood type or blood transfusion refusal. In that case, severely conservative fluid management with the sacrifice of renal functions and hydrocortisone therapy should be considered for better oxygenation.
\end{abstract}

\section{K E Y W O R D S}

AKI, ARDS, COVID-19, ECMO, hydrocortisone, rhesus

\section{1 | INTRODUCTION}

A 62-year-old man with rare blood type exhibited severe respiratory failure due to novel coronavirus. Extracorporeal membrane oxygenation was inapplicable, which requires numerous blood products with the same blood type. We thoroughly restricted fluid volumes by sacrificing renal functions. He was discharged with complete recovery of respiratory and renal functions.
Coronavirus disease 2019 (COVID-19) remains a major threat to the global population since first reported in Wuhan, China. Recent epidemiological studies have shown poor prognosis in patients with COVID-19-related acute respiratory distress syndrome (ARDS). ${ }^{1-3}$ In fact, the mortality rate of COVID-19 patients with mechanical ventilation is reported to be approximately $40 \%-60 \% .^{3-5}$ For the effective management of critically ill COVID-19 patients, the appropriate intervention to respiratory failure 
is crucial. ${ }^{6,7}$ Moreover, if the oxygenation worsens despite mechanical ventilation, extracorporeal membrane oxygenation (ECMO) should be considered, as recommended in some guidelines. ${ }^{8,9}$

Rhesus ( $\mathrm{Rh}$ ) factor is an inherited protein found on the surface of the red blood cells in the body. Since their discovery in the 1940s, it has been considered a specific type of blood type. ${ }^{10}$ In Asian countries, $<1 \%$ of the population has an Rh-negative blood type ${ }^{11}$; therefore, the medical services available for these individuals could be partially limited, e.g., during transfusion and pregnancy. ${ }^{12,13}$ In particular, ECMO is unreasonable in rare blood type patients because it requires a large number of blood products with the same blood type. Thus, if rare blood type patients develop ARDS due to COVID-19, it is crucial to prioritize lung protection. Here, we report the case of an Rh-negative patient with COVID-19-related ARDS who was successfully treated.

\section{2 | CASE PRESENTATION}

A 62-year-old man (height, $165 \mathrm{~cm}$; body weight, $48 \mathrm{~kg}$; body mass index, 17.6) presented with common cold symptoms such as sore throat and fever and visited a local clinic. He undertook a polymerase chain reaction (PCR) test for severe acute respiratory syndrome coronavirus 2 (SARS-CoV-2), which came out positive. Subsequently, the patient was transferred to our emergency department for dyspnea.

Upon arrival at our hospital, although he had clear consciousness (Glasgow Coma Scale of 15) and stable hemodynamics (heart rate, 72 b.p.m.; blood pressure, $150 / 88 \mathrm{mmHg}$ ), his body temperature was high at $38.2^{\circ} \mathrm{C}$. $\mathrm{He}$ also had a respiratory failure with an oxygen saturation of $95 \%$ under an oxygen mask at $5 \mathrm{~L} / \mathrm{min}$. A chest computed tomography $(\mathrm{CT})$ scan revealed bilateral groundglass opacities (GGO) (Figure 1A) with the parenchymal bands in the right lower lobe (Figure 1B), while his blood test revealed severe inflammation and Rh-negative blood type (Table S1). Thus, considering the positive result of his
PCR test in the previous clinic, we diagnosed him with COVID-19 pneumonia.

After being admitted to the depressurized room in our ICU, his oxygenation and hemodynamics deteriorated rapidly (Figure 2A). We initiated mechanical ventilation and administered vasopressors, favipiravir, and ciclesonide. It was impractical to perform ECMO with Rh-negative blood products; therefore, we prioritized lung function protection. For this purpose, we severely restricted the amount of fluid volume, while trying to maintain the bare minimum urine output and blood pressure. In fact, as shown in Figure 2B, the average daily fluid intake was about $2000-3000 \mathrm{ml} /$ day during the first 7 days of hospitalization.

Furthermore, the hypotension was refractory to vasopressors, although the stroke volume variance was maintained within the normal limits. To stabilize the hemodynamics and avoid more fluid infusion, we replaced hydrocortisone $200 \mathrm{mg} /$ day for possible secondary adrenal insufficiency. Thereafter, we could partially taper the amount of vasopressors. Moreover, his oxygenation level also improved, although his $\mathrm{PaO} 2 / \mathrm{FiO} 2$ ratio temporarily dropped to approximately 125 , which is the indication criteria of ECMO for COVID-19-related ARDS. ${ }^{14}$ Then, we gradually reduced the dose of hydrocortisone until discharge because the hemodynamics collapsed in a short while after suspending the hydrocortisone.

In contrast, his serum creatinine level continued to be elevated owing to lower fluid administration and severe systemic inflammation, and it progressed to anuria. Thus, we performed either peritoneal dialysis or continuous hemodiafiltration with a polysulfone membrane from day 7 , depending on the preparation status for renal replacement therapy (RRT) at the time. Thereafter, as the inflammation and body temperature improved over time, the urine output and the $\mathrm{P} / \mathrm{F}$ ratio also gradually normalized. After confirming a negative PCR test result for SARS-CoV-2, he was extubated on day 26 . Finally, on day 73 , he was discharged from our hospital with a full recovery of his respiratory and renal functions, with a serum creatinine level of $0.94 \mathrm{mg} / \mathrm{dl}$.

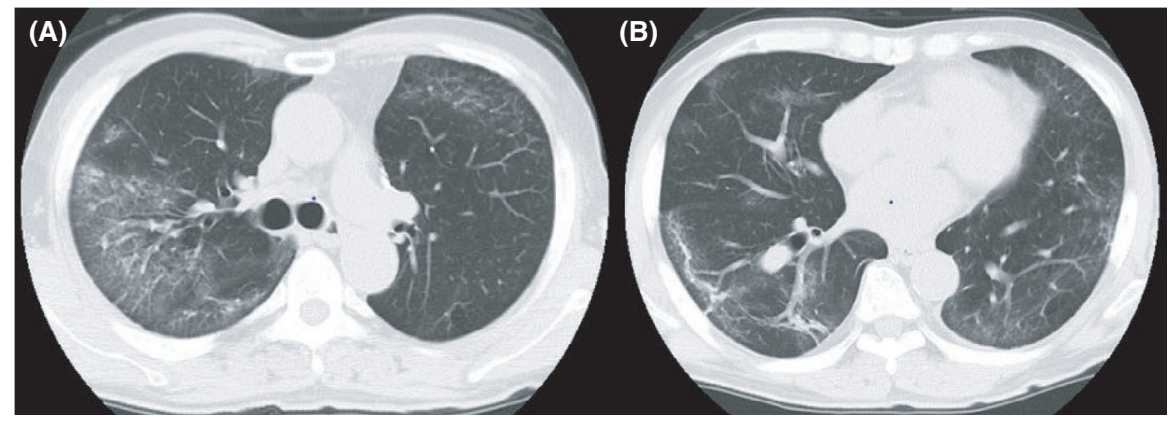

FIG URE 1 Chest computed tomography images on admission. (A) Bilateral ground-glass opacities. (B) Parenchymal bands in the right lower lobe 
FIG URE 2 Clinical course of this case. (A) Chest radiographs on day 1, 6, 13, and 21. (B) Summary of the clinical course
(A)

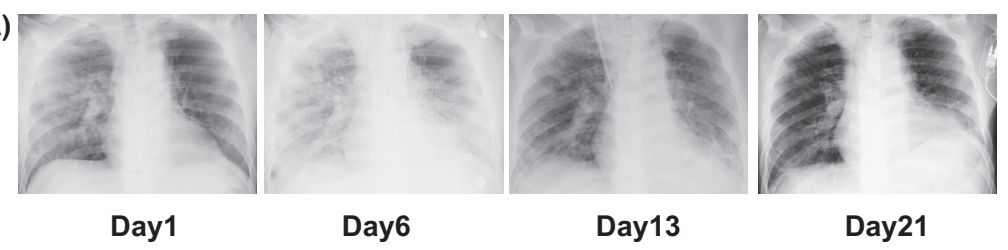

(B)

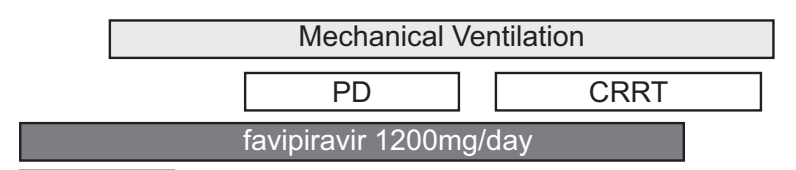

\section{Ciclesonide}

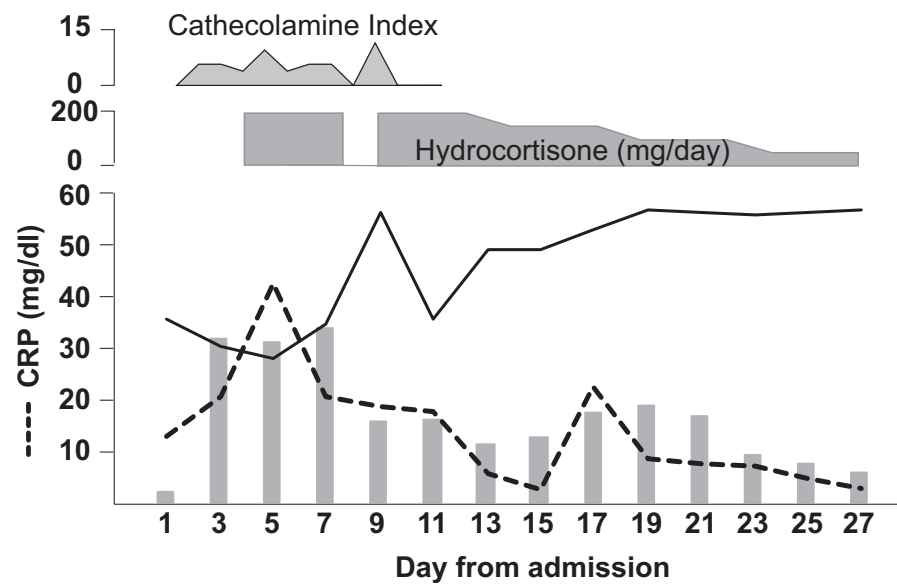

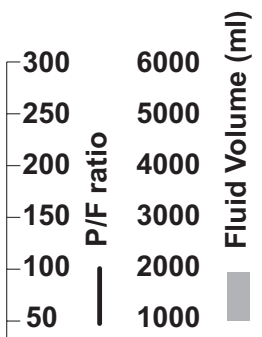

\section{DISCUSSION}

Several studies have shown that the prognosis of COVID19-related ARDS is unfavorable. ${ }^{1-5}$ Furthermore, as Rhnegative is quite a rare blood type, ECMO could not be applied to this COVID-19 patient with Rh-negative blood type because it is unreasonable to ensure enough blood products with the same blood type. Thus, we severely restricted the volume of infusion and administered favipiravir and ciclesonide. Despite the presence of acute kidney injury and hypotension, we managed these complications using hydrocortisone and two types of renal replacement therapy. Finally, the patient survived and was discharged from the hospital.

Some clinical guidelines on ARDS recommend conservative fluid management, aiming to achieve a negative balance in body fluid management. ${ }^{15,16}$ In fact, the benefit of conservative fluid strategy has been supported in the recent randomized controlled trials (RCT) ${ }^{17,18}$ In the conservative group of these RCTs, the total amount of fluid used in the fluid therapy was 3,000-5,000 ml/day. Meanwhile, in our case, the average total fluids from days $2-7$ were $2,942 \mathrm{ml} /$ day. These results indicate that in order to prioritize minimum lung damage, we severely restricted the volume of fluid therapy. We believe that this severe conservative management would enable the successful treatment of respiratory failure without ECMO, although it might partially induce acute kidney injury and refractory hypotension.
As for corticosteroids, there are pros and cons of hydrocortisone administration for critically ill COVID-19 patients. ${ }^{19}$ In fact, the US National Institutes of Health guidelines mentioned in the earlier editions that the efficacy of hydrocortisone was not been robustly supported as that of dexamethasone. The reason for this ambiguous statement could be that the two recent RCTs did not demonstrate the efficacy of hydrocortisone, although a significant reduction in the mortality rate has been reported with the use of dexamethasone. ${ }^{20-22}$ However, we believe that hydrocortisone should be positively considered for severe COVID-19 patients who are suffering from refractory hypotension due to secondary adrenal insufficiency, like our case. In such cases, hydrocortisone could provide two beneficial effects: suppressing the extreme inflammatory response and raising the blood pressure. In the latter RCT, the hydrocortisone group, which was given dependent on the vasopressor requirements, showed the lowest mortality rate, although with no statistical significance. ${ }^{20}$ In fact, in our case, although the purpose of hydrocortisone was to improve the hemodynamics without excessive fluid overload, the oxygenation was also restored rapidly in a short while after administration.

The present treatment strategy for COVID-19 has some limitations. Particularly, we emphasize that this strategy should be applied to patients with rare blood types, blood transfusion refusal, or ECMO contraindications. However, in the common cases of COVID-19 patients with ARDS, 
ECMO should be taken into consideration, as some prominent guidelines suggest. ${ }^{8,9}$

Thus, this case highlights the importance of severely conservative fluid management and hydrocortisone therapy for COVID-19 patients, particularly for those with Rhnegative blood type and secondary adrenal insufficiency. These medical interventions need to be positively considered for COVID-19 patients with rare blood types.

\section{ACKNOWLEDGEMENTS}

We express our deep appreciation to all the staff in the Emergency-Stroke ICU, ICU, and the emergency department of Kyoto University Hospital.

\section{CONFLICT OF INTEREST}

No author has a direct conflict of interest that is relevant to this study.

\section{AUTHOR CONTRIBUTIONS}

$\mathrm{HY}, \mathrm{SO}, \mathrm{NM}$, and KS wrote the manuscript and treated the patient. YK, NT, SK, II, and TT treated the patient. TM supervised the treatment of acute kidney injury. MI, GK, and HD supervised the whole treatment. MK: supervised the treatment on infection control.

\section{ETHICAL APPROVAL}

The Ethics Committee of Kyoto University Graduate School and Faculty of Medicine approved the protocol (R2647).

\section{PREVIOUS PUBLICATIONS}

The part of this case has already been published, focusing on the modality of renal replacement therapy. ${ }^{23}$

\section{CONSENT}

Informed consent was obtained from the patient for publication of this case report.

\section{DATA AVAILABILITY STATEMENT}

The data that support the findings of this study are available on request from the corresponding author. The data are not publicly available due to privacy or ethical restrictions.

\section{ORCID}

Hiroyuki Yamada (1) https://orcid. org/0000-0002-5167-3147

\section{REFERENCES}

1. Wang D, Hu B, Hu C, et al. Clinical characteristics of 138 hospitalized patients with 2019 novel coronavirus-infected pneumonia in Wuhan, China. JAMA. 2020;323:1061-1069.

2. Grasselli G, Zangrillo A, Zanella A, et al. Baseline characteristics and outcomes of 1591 patients infected with SARS-CoV-2 admitted to ICUs of the Lombardy region, Italy. JAMA. 2020;323:1574-1581.

3. Petrilli CM, Jones SA, Yang J, et al. Factors associated with hospital admission and critical illness among 5279 people with coronavirus disease 2019 in New York City: prospective cohort study. BMJ. 2020;369:m1966.

4. Docherty AB, Harrison EM, Green CA, et al. Features of 20133 UK patients in hospital with covid-19 using the ISARIC WHO clinical characterisation protocol: prospective observational cohort study. BMJ. 2020;369:m1985.

5. Yang X, Yu Y, Xu J, et al. Clinical course and outcomes of critically ill patients with SARS-CoV-2 pneumonia in Wuhan, China: a single-centered, retrospective, observational study. Lancet Respir Med. 2020;8:475-481.

6. Matthay MA, Aldrich JM, Gotts JE. Treatment for severe acute respiratory distress syndrome from COVID-19. Lancet Respir Med. 2020;8:433-434

7. Yamakawa K, Yamamoto R, Ishimaru G, et al. Japanese Rapid/ Living recommendations on drug management for COVID-19. Acute Med Surg. 2021;8:e664.

8. Shekar K, Badulak J, Peek G, et al. Extracorporeal life support organization coronavirus disease 2019 interim guidelines: a consensus document from an international group of interdisciplinary extracorporeal membrane oxygenation providers. ASAIO J. 1992;2020(66):707-721.

9. Alhazzani W, Møller MH, Arabi YM, et al. Surviving sepsis campaign: guidelines on the management of critically ill adults with coronavirus disease 2019 (COVID-19). Intensive Care Med. 2020;46:854-887.

10. Landsteiner K, Wiener AS. Studies on an agglutinogen (Rh) in human blood reacting with anti-rhesus sera and with human isoantibodies. J Exp Med. 1941;74:309-320.

11. Fujita Y, Tanaka K, Tanimura M. The distribution of the $\mathrm{Rh}(\mathrm{D})$ blood types in Japan. Jinrui Idengaku Zasshi. 1978;23:197-209.

12. Joy SD, Rossi KQ, Krugh D, O’Shaughnessy RW. Management of pregnancies complicated by anti-E alloimmunization. Obstet Gynecol. 2005;105:24-28.

13. Dunbar NM, Katus MC, Freeman CM, Szczepiorkowski ZM. Easier said than done: ABO compatibility and D matching in apheresis platelet transfusions. Transfusion. 2015;55: 1882-1888.

14. Galvagno S, Bennett S. Indications for ECMO for ICU COVID-19 patients. https://www.asahq.org/-/media/sites/asahq/files/ public/about-asa/governance-and-committees/caesar-covid/ indications-for-ecmo-for-icu-covid-19-patients.pdf. Accessed September 1, 2021.

15. Hashimoto S, Sanui M, Egi M, et al. The clinical practice guideline for the management of ARDS in Japan. J Intensive Care. 2017;5:50.

16. Griffiths MJD, McAuley DF, Perkins GD, et al. Guidelines on the management of acute respiratory distress syndrome. BMJ Open Respir Res. 2019;6:e000420.

17. Wiedemann HP, Wheeler AP, Bernard GR, et al. Comparison of two fluid-management strategies in acute lung injury. $N$ Engl $J$ Med. 2006;354:2564-2575.

18. Chen C, Kollef MH. Targeted fluid minimization following initial resuscitation in septic shock: a pilot study. Chest. 2015;148:1462-1469. 
19. Berton AM, Prencipe N, Giordano R, Ghigo E, Grottoli S. Systemic steroids in patients with COVID-19: pros and contras, an endocrinological point of view. J Endocrinol Invest. 2021;44(4):873-875.

20. Angus DC, Derde L, Al-Beidh F, et al. Effect of hydrocortisone on mortality and organ support in patients with severe COVID-19: the REMAP-CAP COVID-19 corticosteroid domain randomized clinical trial. JAMA. 2020;324:1317-1329.

21. Dequin PF, Heming N, Meziani F, et al. Effect of hydrocortisone on 21-day mortality or respiratory support among critically Ill patients with COVID-19: a randomized clinical trial. JAMA. 2020;324:1298-1306.

22. Horby P, Lim WS, Emberson JR, et al. Dexamethasone in hospitalized patients with Covid-19. $N$ Engl J Med. 2021;384:693-704.
23. Nagatomo M, Yamada H, Shinozuka K, Shimoto M, Yunoki T, Ohtsuru S. Peritoneal dialysis for COVID-19-associated acute kidney injury. Crit Care. 2020;24:309.

\section{SUPPORTING INFORMATION}

Additional supporting information may be found in the online version of the article at the publisher's website.

How to cite this article: Yamada $\mathrm{H}$, Ohtsuru S, Nagatomo M, et al. Successful treatment of COVID19-related acute respiratory distress syndrome with a rare blood type: A case report. Clin Case Rep. 2021;9:e04859. https://doi.org/10.1002/ccr3.4859 